\title{
Factos e mitos sobre envelhecimento em alunos no início de licenciatura em educação social
}

\section{Facts and myths about aging in students at the beginning of a degree in social education}

\author{
Lia Araújo*, Maria João Amante**, Susana Fonseca* \\ * Instituto Politécnico de Viseu, **Universidade do Porto
}

\begin{abstract}
Resumo
Este estudo analisa a percepção que alunos do $1^{\circ}$ ano de uma licenciatura em Educação Social têm acerca das pessoas idosas com base nas respostas de 259 alunos a um questionário sobre factos e mitos do envelhecimento. Os resultados demonstram a existência de ideias erradas na maioria dos participantes, nomeadamente a generalização de que a velhice pode ser encarada como uma segunda infância $(75 \%)$ e que a maioria das pessoas idosas se torna senil durante a velhice (63\%). Justifica-se, assim, um maior investimento em atividades de desmistificação que possibilitem o respeito e a valorização dos mais velhos. Palavras-chave: educação social, idadismo, envelhecimento, mitos, intervenção socioeducativa.
\end{abstract}

\begin{abstract}
This study analyses the perception that 1 st year students of a degree in Social Education have about elderly people, through the answers of 259 students to a questionnaire about facts and myths of aging. The results demonstrate the existence of misconceptions in most participants, namely the generalization that old age can be seen as a second childhood $(75 \%)$ and that the majority of older people become senile during old age $(63 \%)$. These results justify a greater investment in demystification activities that allow the respect and valuation of older people.

Keywords: social education, ageism, aging, myths, socioeducative intervention.
\end{abstract}

O envelhecimento da população é incontornavelmente um dos grandes desafios transversais a todo o século XXI. Em Portugal, segundo um recente exercício de projeções de população residente (INE, 2017), espera-se o agravamento da diminuição da população jovem e do aumento da população idosa. O Instituto Nacional de Estatística estima que, entre 2015 e 2080, a população com 65 ou mais anos de idade residente em Portugal passe de 2.1 para 2.8 milhões de pessoas, esperando-se uma duplicação do índice de envelhecimento, de 147 para 317 pessoas idosas por cada 100 jovens, em 2080 (INE, 2017)

Este cenário global de envelhecimento demográfico terá muitas implicações sociais e individuais que carecem de atenção e antecipação. Este é o alerta dado por várias entidades, nomeadamente pela Organização
Mundial de Saúde, que sublinha a importância de ações em prol do envelhecimento positivo serem uma prioridade de vários sectores para além do da saúde (WHO, 2014). A nível social, o apoio e a capacitação das pessoas mais velhas, nomeadamente através da sua participação e envolvimento na comunidade, são estratégias que imperam e que requerem de profissionais preparados para estes desafios. Rosen, Zlotnik e Singer (2003) a este propósito, referem a importância de "gerontolizar" a educação dos trabalhadores sociais e de os orientar para esta área de conhecimento e intervenção.

A formação inicial, correspondente ao $1^{\circ}$ ciclo, tem-se revelado como decisiva no desenvolvimento de conhecimentos e de competências para a intervenção com pessoas idosas mas também no desenvolvimento de interesse dos estudantes por esta área de trabalho (Boswell, 2012). No entanto, investigações têm vindo a demonstrar que estudantes de ensino superior apresentam atitudes negativas e idadistas relativamente aos mais velhos. O idadismo, definido por Butler (1980) como o processo de discriminação com base na idade, está associado ao não reconhecimento das capacidades e do potencial das pessoas idosas (Fernández-Ballesteros, 2013). Apesar de ser uma atitude social relativamente generalizada, o idadismo tem sido explorado em cursos de formação de profissionais que irão intervir com pessoas idosas (Coffey et al., 2015). Na sua maioria, os resultados são concordantes e apontam para a existência de estereótipos, que associam a velhice a dependência, tristeza, solidão, incompetência e doença (Vicente \& Afonso, 2012). Estas atitudes negativas influenciam as escolhas de carreira, bem como a qualidade dos cuidados prestados por estes futuros profissionais (Gething et al., 2002). Dos vários estudos apresentados na revisão da literatura de Coffey e colegas (2015) e da investigação em contexto Português realizada por Gonçalves e colaboradores (2011), apenas se conhecem as atitudes de estudantes dos cursos de enfermagem, psicologia e serviço social.

A Educação Social tem-se vindo a assumir como área de intervenção fulcral na promoção do envelhecimento ativo, i.e., na otimização das oportunidades para a saúde, participação, segurança e educação ao longo da vida, 
para melhorar a qualidade de vida das pessoas que envelhecem (ILC-Brazil, 2015, Araújo, 2014). Assim, por se considerar necessário conhecer os conhecimentos, percepções e atitudes dos futuros profissionais de Educação Social, o presente estudo analisa as imagens sociais que alunos do $1^{\circ}$ ano de uma licenciatura em Educação Social têm acerca das pessoas idosas.

\section{Método}

Ao longo dos últimos 5 anos letivos (2012-2017), alunos do primeiro ano do curso de Licenciatura em Educação Social de um Instituto Politécnico Português, no âmbito de uma unidade curricular sobre Psicologia do Adulto e Idoso, responderam a um questionário de verdadeiro e falso sobre mitos do envelhecimento. As questões foram definidas com base no Palmore Facts on Aging Quiz, o qual é reconhecido como o instrumento mais utilizado para avaliar conhecimentos gerais, factos e mitos sobre a fase avançada de vida (Palmore, 1977). O questionário foi utilizado na primeira aula da referida unidade curricular, antes da lecionação dos respetivos conteúdos programáticos. No total, responderam 259 alunos, sendo 58 do ano letivo 2012/2013, 52 de 2013/2014, 53 de 2014/2015, 47 de 2015/2016, e 49 de 2016/2017. As respostas foram analisadas, comparando-se as diferenças entre anos letivos, com recurso ao Statistical Package for the Social Sciences (SPSS, versão 22), tendo sido considerado o valor de $\mathrm{p}<0.05$ como significativo.

\section{Resultados}

Os resultados demonstram a existência de ideias erradas acerca do processo de envelhecimento na maioria dos participantes. Na tabela 2 é possível verificar o número de alunos que responderam como "verdadeiro" aos mitos do questionário (Tabela 1 ).

Os mitos em que se verificou um maior nível de concordância, com mais de 150 alunos a responder afirmativamente, foram $A$ maior parte das pessoas idosas tende a estar preocupada com a morte $(\mathrm{n}=208), A$ velhice pode ser encarada como uma segunda infância $(\mathrm{n}=194)$, Os sentimentos de solidão produzem-se quando não há família elou as pessoas vivem sozinhas $(\mathrm{n}=194)$, A maior parte das pessoas idosas vive num limiar abaixo da pobreza $(\mathrm{n}=189), \quad$ A personalidade altera-se fortemente com a idade ( $\mathrm{n}=166)$, Um idoso é todo aquele que tem 65 anos ou mais de vida $(\mathrm{n}=160)$ e A depressão é um problema muito comum na velhice $(\mathrm{n}=158)$.

Verificam-se diferenças estatisticamente significativas entre anos letivos para a maioria das questões consideradas. A este propósito, e considerando as três primeiras questões (Q1, Q2 e Q3), verifica-se que a maioria dos respondentes concorda com as afirmações, como exceção dos alunos do ano letivo 2016/2017 em que um menor número $(\mathrm{n}=22,44.9 \%)$ respondeu afirmativamente $(\mathrm{p}<0.05)$. Também nas questões $6,7,8$, 13 e 14, foi no último ano letivo em que se verificou uma menor concordância com as questões apresentadas. Já na questão 5, é no ano letivo 2014/2015 que se verifica uma menor percentagem $(32.1 \%)$ de alunos que concordam com a afirmação $(\mathrm{p}<0.05)$.
No caso da questão 10 (A maioria das pessoas idosas diz que está feliz a maior parte do tempo), onde seria expectável uma resposta afirmativa, 77 alunos (29.7\%) responderam sim. Apenas no ano letivo 2014/2015 uma percentagem maior e razoável $(67.9 \%)$ respondeu que $\operatorname{sim}(\mathrm{p}<0.05)$.

Pela positiva, verificou-se que um menor número de respondentes considera que as pessoas idosas são incapazes de aprender novas informações $(\mathrm{n}=9 ; 3.5 \%) \mathrm{e}$ que raramente alguém depois dos 65 anos produz um trabalho de grande mérito e reconhecimento $(\mathrm{n}=97$; $37.5 \%)$.

Tabela 1.

Questões sobre envelhecimento

\begin{tabular}{|c|c|}
\hline Q1 & $\begin{array}{l}\text { Um idoso é todo aquele que tem } 65 \text { anos ou mais de } \\
\text { vida }\end{array}$ \\
\hline Q2 & A personalidade altera-se fortemente com a idade \\
\hline Q3 & A depressão é um problema muito comum na velhice \\
\hline Q4 & $\begin{array}{l}\text { Os sentimentos de solidão produzem-se quando não há } \\
\text { família e/ou as pessoas vivem sozinhas }\end{array}$ \\
\hline Q5 & $\begin{array}{l}\text { A maioria das pessoas idosas torna-se senil durante a } \\
\text { velhice }\end{array}$ \\
\hline Q6 & $\begin{array}{l}\text { Com a idade perde-se a capacidade para se ter relações } \\
\text { sexuais }\end{array}$ \\
\hline Q7 & $\begin{array}{l}\text { A maior parte das pessoas idosas tende a estar } \\
\text { preocupada com a morte }\end{array}$ \\
\hline Q8 & $\begin{array}{l}\text { A velhice pode ser encarada como uma segunda } \\
\text { infância }\end{array}$ \\
\hline Q9 & Durante a velhice é difícil prevenir doencas \\
\hline Q10 & $\begin{array}{l}\text { A maioria das pessoas idosas diz que está feliz a maior } \\
\text { parte do tempo }\end{array}$ \\
\hline Q11 & $\begin{array}{l}\text { As pessoas idosas são incapazes de aprender novas } \\
\text { informacões }\end{array}$ \\
\hline Q12 & $\begin{array}{l}\text { As pessoas idosas parecem-se mais entre si que as } \\
\text { jovens }\end{array}$ \\
\hline Q13 & A velhice deve ser um período de descanso \\
\hline Q14 & $\begin{array}{l}\text { A maior parte das pessoas idosas vive num limiar } \\
\text { abaixo da pobreza }\end{array}$ \\
\hline Q15 & $\begin{array}{l}\text { Raramente alguém depois dos } 65 \text { anos produz um } \\
\text { trabalho de grande mérito e reconhecimento }\end{array}$ \\
\hline
\end{tabular}


Tabela 2.

Respostas afirmativas aos mitos do envelhecimento

\begin{tabular}{|c|c|c|c|c|c|c|}
\hline & $\begin{array}{c}12 / 13 \\
\mathrm{n} \\
(\%)\end{array}$ & $\begin{array}{c}13 / 14 \\
\mathrm{n} \\
(\%)\end{array}$ & $\begin{array}{c}14 / 15 \\
\mathrm{n} \\
(\%)\end{array}$ & $\begin{array}{c}15 / 16 \\
\mathrm{n} \\
(\%)\end{array}$ & $\begin{array}{c}16 / 17 \\
\mathrm{n} \\
(\%)\end{array}$ & $\begin{array}{c}\text { Total } \\
\mathrm{n} \\
(\%)\end{array}$ \\
\hline Q1 & $\begin{array}{c}45 \\
(77.6)\end{array}$ & $\begin{array}{c}36 \\
(69.2)\end{array}$ & $\begin{array}{c}31 \\
(60.8)\end{array}$ & $\begin{array}{c}26 \\
(55.3)\end{array}$ & $\begin{array}{c}22 \\
(44.9)\end{array}$ & $\begin{array}{c}160^{*} \\
(61.8)\end{array}$ \\
\hline Q2 & $\begin{array}{c}49 \\
(84.5)\end{array}$ & $\begin{array}{c}35 \\
(67.3)\end{array}$ & $\begin{array}{c}31 \\
(58.5)\end{array}$ & $\begin{array}{c}29 \\
(61.7)\end{array}$ & $\begin{array}{c}22 \\
(44.9)\end{array}$ & $\begin{array}{c}166^{*} \\
(64.1)\end{array}$ \\
\hline Q3 & $\begin{array}{c}39 \\
(67.2)\end{array}$ & $\begin{array}{c}37 \\
(74.0)\end{array}$ & $\begin{array}{c}34 \\
(64.2)\end{array}$ & $\begin{array}{c}26 \\
(55.3)\end{array}$ & $\begin{array}{c}22 \\
(44.9)\end{array}$ & $\begin{array}{c}158^{*} \\
(61.0)\end{array}$ \\
\hline Q4 & $\begin{array}{c}51 \\
(87.9)\end{array}$ & $\begin{array}{c}45 \\
(86.5)\end{array}$ & $\begin{array}{c}31 \\
(58.5)\end{array}$ & $\begin{array}{c}36 \\
(76.6)\end{array}$ & $\begin{array}{c}31 \\
(64.6)\end{array}$ & $\begin{array}{c}194^{*} \\
(74.9)\end{array}$ \\
\hline Q5 & $\begin{array}{c}27 \\
(87.1)\end{array}$ & $\begin{array}{c}36 \\
(69.2)\end{array}$ & $\begin{array}{c}17 \\
(32.1)\end{array}$ & $\begin{array}{c}38 \\
(80.9)\end{array}$ & $\begin{array}{c}27 \\
(55.1)\end{array}$ & $\begin{array}{c}145^{*} \\
(55.9)\end{array}$ \\
\hline Q6 & $\begin{array}{c}36 \\
(62.1)\end{array}$ & $\begin{array}{c}31 \\
(59.6)\end{array}$ & $\begin{array}{c}25 \\
(47.2)\end{array}$ & $\begin{array}{c}24 \\
(51.1)\end{array}$ & $\begin{array}{c}18 \\
(36.7)\end{array}$ & $\begin{array}{c}134 \\
(51.7)\end{array}$ \\
\hline Q7 & $\begin{array}{c}46 \\
(79.3)\end{array}$ & $\begin{array}{c}42 \\
(80.8)\end{array}$ & $\begin{array}{c}50 \\
(94.3)\end{array}$ & $\begin{array}{c}41 \\
(89.1)\end{array}$ & $\begin{array}{c}29 \\
(59.2)\end{array}$ & $\begin{array}{c}208^{*} \\
(80.3)\end{array}$ \\
\hline Q8 & $\begin{array}{c}49 \\
(84.5)\end{array}$ & $\begin{array}{c}39 \\
(75.0)\end{array}$ & $\begin{array}{c}45 \\
(84.9)\end{array}$ & $\begin{array}{c}34 \\
(72.3)\end{array}$ & $\begin{array}{c}27 \\
(55.1)\end{array}$ & $\begin{array}{c}194 * \\
(74.9)\end{array}$ \\
\hline Q9 & $\begin{array}{c}36 \\
(62.1)\end{array}$ & $\begin{array}{c}22 \\
(42.3)\end{array}$ & $\begin{array}{c}25 \\
(47.2)\end{array}$ & $\begin{array}{c}17 \\
(36.2)\end{array}$ & $\begin{array}{c}25 \\
(52.1)\end{array}$ & $\begin{array}{c}125 \\
(48.3)\end{array}$ \\
\hline Q10 & $\begin{array}{c}7 \\
(12.1)\end{array}$ & $\begin{array}{c}8 \\
(15.4)\end{array}$ & $\begin{array}{c}36 \\
(67.9)\end{array}$ & $\begin{array}{c}11 \\
(23.9)\end{array}$ & $\begin{array}{c}15 \\
(31.3)\end{array}$ & $\begin{array}{c}77 * \\
(29.7)\end{array}$ \\
\hline Q11 & $\begin{array}{c}2 \\
(3.4)\end{array}$ & $\begin{array}{c}6 \\
(11.8)\end{array}$ & $\begin{array}{c}0 \\
(0.0)\end{array}$ & $\begin{array}{c}1 \\
(2.1)\end{array}$ & $\begin{array}{c}0 \\
(0.0)\end{array}$ & $\begin{array}{c}9 * \\
(3.5)\end{array}$ \\
\hline Q12 & $\begin{array}{c}18 \\
(58.1)\end{array}$ & $\begin{array}{c}34 \\
(66.7)\end{array}$ & $\begin{array}{c}30 \\
(56.6)\end{array}$ & $\begin{array}{c}32 \\
(68.1)\end{array}$ & $\begin{array}{c}28 \\
(59.6)\end{array}$ & $\begin{array}{c}142 \\
(54.8)\end{array}$ \\
\hline Q13 & $\begin{array}{c}32 \\
(55.2)\end{array}$ & $\begin{array}{c}23 \\
(45.1)\end{array}$ & $\begin{array}{c}27 \\
(50.9)\end{array}$ & $\begin{array}{c}25 \\
(53.2)\end{array}$ & $\begin{array}{c}22 \\
(44.9)\end{array}$ & $\begin{array}{c}129 \\
(49.8)\end{array}$ \\
\hline Q14 & $\begin{array}{c}51 \\
(87.9)\end{array}$ & $\begin{array}{c}34 \\
(65.4)\end{array}$ & $\begin{array}{c}34 \\
(65.4)\end{array}$ & $\begin{array}{c}40 \\
(87.0)\end{array}$ & $\begin{array}{c}30 \\
(61.2)\end{array}$ & $\begin{array}{c}189 * \\
(72.9)\end{array}$ \\
\hline Q15 & $\begin{array}{c}18 \\
(31.0)\end{array}$ & $\begin{array}{c}22 \\
(42.3)\end{array}$ & $\begin{array}{c}16 \\
(30.8)\end{array}$ & $\begin{array}{c}18 \\
(38.3)\end{array}$ & $\begin{array}{c}23 \\
(46.9)\end{array}$ & $\begin{array}{c}97 \\
(37.5)\end{array}$ \\
\hline
\end{tabular}

\section{Discussão}

Os resultados apresentados revelam a existências de conceitos errados e estereótipos acerca do envelhecimento e das pessoas idosas na maioria dos inquiridos. Apesar da frequência elevada de estereótipos ser transversal aos alunos de todo o período analisado, verifica-se uma diminuição nos últimos anos letivos, com diferenças estatisticamente significativas, o que pode estar relacionado com o aumento da informação e de imagens de envelhecimento positivo que se tem difundido, nomeadamente através dos meios de comunicação social (Ferreira, Bianchi, Menegócio, \& Zago, 2014).

Visto que os alunos participantes no presente estudo serão futuros profissionais de Educação Social que, com probabilidade crescente, poderão trabalhar com pessoas idosas, é fulcral promover uma visão realista e positiva da fase avançada de vida. As instituições de Ensino Superior, responsáveis pela formação e capacitação dos futuros profissionais, têm uma responsabilidade de peso neste sentido. Estudos internacionais suportam esta ideia ao demonstrar que a existência de currículos com conteúdos na área do envelhecimento correlaciona-se com maior conhecimento dos assuntos que afetam a fase avançada de vida, bem como com o nível de interesse dos alunos em trabalhar com pessoas idosas (Hughes \& Heycox, 2006). Cummings, Galambos e Decoster.
(2003) referem que também o contacto direto com os mais velhos, em contexto de sala de aula e em contexto prático, deve ser promovido.

Neste sentido, sugere-se o estudo das características biopsicossociais do processo de envelhecimento, com ênfase nos modelos de envelhecimento ativo e bem sucedido nos cursos de Intervenção Social. A este propósito, Cozort (2008) acredita que as Escolas beneficiariam de investigações junto dos alunos para conhecer as suas perceções e atitudes acerca do envelhecimento, cujos resultados poderiam contribuir para ajudar os alunos a conhecer melhor as forças e dificuldades do processo de envelhecimento e os docentes a melhorar o currículo.

Já na sociedade, parece ser necessário um maior investimento em atividades de desmistificação que possibilitem o respeito e a valorização dos mais velhos (Marques, 2011).

Ao nível da modificação de atitudes, estereótipos, mitos e preconceitos sobre os mais velhos, muitas vezes associados a discriminação, gerontofobia e atitudes de infantilização (Berger \& Mailloux-Poirier, 1994), é de destacar o papel do Educador Social. Atividades no âmbito da gerontologia educativa, que incluam a sensibilização e educação a públicos idosos mas também ações dirigidas à sociedade em geral ou a grupos etários e sociais muito específicos, podem contribuir para proporcionar um maior conhecimento da realidade da velhice e, neste sentido, desenvolver uma imagem social mais verdadeira, dinâmica e ativa (Bermejo, 2005). Também os projetos intergeracionais são particularmente interessantes neste âmbito, pois através de atividades em que os mais velhos possam demonstrar as suas capacidades e saberes ao mesmo tempo que aprendem ferramentas dominadas pelas gerações mais novas, podem promover a quebra de barreiras entre gerações, nomeadamente nas tecnologias e informação (Ferreira et al., 2014) e aumentar a sua participação na sociedade (Araújo, 2014).

\section{Referências}

Araújo, L. (2014). A participação social das pessoas idosas. In S. Azevedo \& F. Correia (Coords), Educação e integração social - Livro de Actas do 3. ${ }^{\circ}$ Congresso Internacional de Educação Social (pp. 91-106). Porto: APTSES, Fronteira do Caos.

Berger, L. \& Mailloux-Poirier, D. (1994). Pessoas Idosas: Uma abordagem global. Lisboa: Lusodidacta.

Bermejo, L. (2005).Gerontología Educativa: Cómo disenar proyectos educativos com personas mayores. Madrid: Médica Panamericana.

Boswell, S. (2012). 'Old people are cranky': helping professional trainees' knowledge, attitudes, aging anxiety, and interest in working with older adults. Educational Gerontology, 38, 465-472.

Butler, R. N. (1980). Ageism: a foreword. Journal of Social Issues, 36(2), 8-11.

Cummings, S., Galambos, C., \& Decoster V. (2003). Predictors of MSW employment in gerontological practice. Educational Gerontology, 29, 295-312. 
Coffey, A., Buckley, C., Gaidys, U., Sasoni, J., Arola, M., ... \& Tyrrell, M. (2015). Beliefs of students about growing older and perceptions of working in gerontology. Nursing Older People, 27, 33-37.

Cozort, R. (2008). Student nurses' attitudes regarding older adults: Strategies for fostering improvement through academia. Teaching and Learning in Nursing, 3, 21-25.

Fernández-Ballesteros, R. (2013). Possibilities and Limitations of Age. In A. Oliveira (Coord.), Promoting Conscious and Active Learning and Aging How to Face Current and Future Challenges? (pp. 25-74). Coimbra: Imprensa da Universidade de Coimbra.

Ferreira, M.G., Bianchi, M., Menegócio, A.M.M., Zago, G.M. (2014). Desconstruindo a imagem do idoso nos meios midiáticos. Revista Kairós Gerontologia, 17(4), 211-223.

Gething, L., Fethney, J., McKee, K., Goff, M., Churchward, M., \& Matthews, S. (2002). Knowledge, stereotyping and attitudes towards self ageing. Australasian Journal on Ageing, 21(2), 64-79.

Gonçalves, D., Guedes, J., Fonseca, A.M., Pinto, F., Martín, I., Byrne, G., \& Pachana, N. (2011). Attitudes, knowledge, and interest: preparing university students to work in an aging world. International Psychogeriatrics, 23, 315-321.

Hughes, M., \& Heycox, K. (2006). Knowledge and interest in ageing: a study of 6nal-year social work students. Australasian Journal on Ageing, 25(2), 94-96.

ILC-Brazil, International Longevity Centre Brazil (2015). Active Ageing: A Policy Framework in Responde to the Longevity Revolution. Rio de Janeiro: ILC-Brazil. Obtido de: http://ilcbrazil.org/wp-content/uploads/2016/02/Activ e-Ageing-A-Policy-Framework-ILC-Brazil_web.pdf

Instituto Nacional de Estatística - INE (2017). Mantém-se o agravamento do envelhecimento demográfico, em Portugal, que só tenderá a estabilizar daqui a cerca de 40 anos. Obtido em: https://www.ine.pt/xportal/xmain?xpid=INE\&xpgid=i ne_destaques\&DESTAQUESdest_boui=277695619\& DESTAQUESmodo $=2$

Marques, S. (2011). Discriminação da Terceira Idade. Lisboa: Fundação Francisco Manuel dos Santos.

Palmore, E. (1977). Facts on aging: A short quiz. The Gerontologist, 17 (4), 315-320.

Rosen, A., Zlotnik J., \& Singer, T. (2003). Basic Gerontological Competence for All Social Workers. Journal of Gerontological Social Work, 39.

Vicente, F., \& Afonso, R.M. (2012). Imagens Do Idoso e do Envelhecimento em Estudantes Universitários. International Journal of Developmental and Educational Psychology, 2, 87-94

World Health Organization (2014).“Ageing well” must be a global priority. Obtido em: http://www.who.int/mediacentre/news/releases/2014/1 ancet-ageing-series/en/

\section{Agradecimentos}

A todos os alunos do curso de Licenciatura em Educação Social que, durante o período contemplado na recolha de dados, participaram na resposta a este questionário. Ao Centro de Estudos em Educação, Tecnologias e Saúde (CI\&DETS) do Instituto Politécnico de Viseu. 